PROCEEDINGS OF THE

AMERICAN MATHEMATICAL SOCIETY

Volume 126, Number 10, October 1998, Pages 3117-3124

S $0002-9939(98) 04605-\mathrm{X}$

\title{
CONTRACTIVE MAPPINGS, KANNAN MAPPINGS AND METRIC COMPLETENESS
}

\author{
NAOKI SHIOJI, TOMONARI SUZUKI, AND WATARU TAKAHASHI
}

(Communicated by Palle E. T. Jorgensen)

\begin{abstract}
In this paper, we first study the relationship between weakly contractive mappings and weakly Kannan mappings. Further, we discuss characterizations of metric completeness which are connected with the existence of fixed points for mappings. Especially, we show that a metric space is complete if it has the fixed point property for Kannan mappings.
\end{abstract}

\section{INTRODUCTION}

Let $X$ be a metric space with metric $d$. Then a function $p$ from $X \times X$ into $[0, \infty)$ is called a $w$-distance on $X$ if it satisfies the following:

(1) $p(x, z) \leq p(x, y)+p(y, z)$ for all $x, y, z \in X$;

(2) $p$ is lower semicontinuous in its second variable;

(3) for each $\varepsilon>0$, there exists $\delta>0$ such that $p(z, x) \leq \delta$ and $p(z, y) \leq \delta$ imply $d(x, y) \leq \varepsilon$.

The concept of a $w$-distance was first introduced by Kada, Suzuki and Takahashi [6]. They give some examples of $w$-distance and improved Caristi's fixed point theorem [2], Ekeland's variational principle [4] and the nonconvex minimization theorem according to Takahashi [12]. We denote by $W(X)$ the set of all $w$-distances on $X$. A mapping $T$ from $X$ into itself is called weakly contractive [11] if there exist $p \in W(X)$ and $r \in[0,1)$ such that

$$
p(T x, T y) \leq r p(x, y) \quad \text { for all } \quad x, y \in X .
$$

In particular, if $p=d, T$ is called contractive. Suzuki and Takahashi [11] proved that a metric space is complete if and only if it has the fixed point property for weakly contractive mappings. A mapping $T$ from $X$ into itself is called weakly Kannan [10] if there exist $p \in W(X)$ and $\alpha \in[0,1 / 2)$ such that

$$
p(T x, T y) \leq \alpha(p(T x, x)+p(T y, y)) \text { for all } x, y \in X,
$$

or

$$
p(T x, T y) \leq \alpha(p(T x, x)+p(y, T y)) \quad \text { for all } \quad x, y \in X .
$$

In particular, if $p=d, T$ is called Kannan [7]. Suzuki [10] proved that a complete metric space has the fixed point property for weakly Kannan mappings. On the

Received by the editors October 25, 1996 and, in revised form, February 27, 1997.

1991 Mathematics Subject Classification. Primary 54E50.

Key words and phrases. Completeness, contractive mapping, Kannan mapping, fixed point, mean. 
other hand, characterizations of metric completeness have been discussed by many authors (cf. $[3,5,8,9,12]$ ). It has been known that the fixed point property for contractive mappings does not characterize metric completeness. For example, see [11]. But $\mathrm{Hu}[5]$ proved that a metric space is complete if every closed subspace has the fixed point property for contractive mappings. Reich [9] also proved that a metric space is complete if every closed subspace has the fixed point property for Kannan mappings. We recall that a mapping $T$ from a metric space $X$ into itself is said to be Caristi if there exists a lower semicontinuous function $\varphi$ from $X$ into $[0, \infty)$ such that $d(x, T x) \leq \varphi(x)-\varphi(T x)$ for all $x \in X$. Note that Caristi mappings include Kannan mappings and contractive mappings. Kirk [8] proved that a metric space is complete if it has the fixed point property for Caristi mappings. Thus Caristi mappings characterize metric completeness whereas contractive mappings do not. This leaves open the question whether Kannan mappings characterize metric completeness or not.

In this paper, we first study the relationship between weakly contractive mappings and weakly Kannan mappings. Further, we discuss characterizations of metric completeness which are connected with the existence of fixed points for mappings. Especially, we show that a metric space is complete if it has the fixed point property for Kannan mappings.

\section{Preliminaries}

Throughout this paper, we denote by $\mathbb{N}, \mathbb{Z}, \mathbb{Q}$ and $\mathbb{R}$ the sets of positive integers, integers, rational numbers and real numbers, respectively.

Let $X$ be a metric space with metric $d$. A $w$-distance $p$ on $X$ is called symmetric if $p(x, y)=p(y, x)$ for all $x, y \in X$. We denote by $W_{0}(X)$ the set of all symmetric $w$-distances on $X$. Note that the metric $d$ is an element in $W_{0}(X)$. We denote by $W C_{1}(X)$ the set of all mappings $T$ from $X$ into itself such that there exist $p \in W(X)$ and $r \in[0,1)$ satisfying

$$
p(T x, T y) \leq \operatorname{rp}(x, y) \text { for all } x, y \in X,
$$

i.e., the set of all weakly contractive mappings from $X$ into itself. We define the sets $W C_{2}(X), W C_{0}(X), W K_{1}(X), W K_{2}(X)$ and $W K_{0}(X)$ of mappings from $X$ into itself as follows: $T \in W C_{2}(X)$ if and only if there exist $p \in W(X)$ and $r \in[0,1)$ such that

$$
p(T x, T y) \leq r p(y, x) \quad \text { for all } \quad x, y \in X
$$

$T \in W C_{0}(X)$ if and only if there exist $p \in W_{0}(X)$ and $r \in[0,1)$ such that

$$
p(T x, T y) \leq r p(x, y) \text { for all } x, y \in X ;
$$

$T \in W K_{1}(X)$ if and only if there exist $p \in W(X)$ and $\alpha \in[0,1 / 2)$ such that

$$
p(T x, T y) \leq \alpha(p(T x, x)+p(T y, y)) \quad \text { for all } \quad x, y \in X ;
$$

$T \in W K_{2}(X)$ if and only if there exist $p \in W(X)$ and $\alpha \in[0,1 / 2)$ such that

$$
p(T x, T y) \leq \alpha(p(T x, x)+p(y, T y)) \text { for all } x, y \in X ;
$$

$T \in W K_{0}(X)$ if and only if there exist $p \in W_{0}(X)$ and $\alpha \in[0,1 / 2)$ such that

$$
p(T x, T y) \leq \alpha(p(T x, x)+p(T y, y)) \quad \text { for all } \quad x, y \in X .
$$

We recall $T$ is weakly Kannan if $T \in W K_{1}(X) \cup W K_{2}(X)$. 
Let $\mu$ be a mean on $\mathbb{N}$, i.e., a continuous linear functional on $l^{\infty}$ satisfying $\|\mu\|=$ $1=\mu(1)$. Then we know that $\mu$ is a mean on $\mathbb{N}$ if and only if $\inf _{n \in \mathbb{N}} a_{n} \leq \mu(a) \leq$ $\sup _{n \in \mathbb{N}} a_{n}$ for every $a=\left(a_{1}, a_{2}, \cdots\right) \in l^{\infty}$. According to time and circumstances, we use $\mu_{n}\left(a_{n}\right)$ instead of $\mu(a)$. A mean on $\mathbb{N}$ is called a Banach limit [1] if $\mu_{n}\left(a_{n}\right)=$ $\mu_{n}\left(a_{n+1}\right)$ for every $a=\left(a_{1}, a_{2}, \cdots\right) \in l^{\infty}$. We also know that if $\mu$ is a Banach limit, then $\underline{\lim }_{n} a_{n} \leq \mu_{n}\left(a_{n}\right) \leq \varlimsup_{n} a_{n}$ for every $a=\left(a_{1}, a_{2}, \cdots\right) \in l^{\infty}$.

\section{Results}

The following two theorems are our main results, which are proved in Section 4.

Theorem 1. Let $X$ be a metric space. Then

$$
W C_{1}(X)=W C_{0}(X)=W K_{1}(X)=W K_{0}(X) \subset W C_{2}(X)=W K_{2}(X) .
$$

Theorem 2. Let $X$ be a metric space with metric $d$. Then the following are equivalent:

(i) $X$ is complete;

(ii) every Kannan mapping $T$ from $X$ into itself has a fixed point in $X$;

(iii) for every bounded sequence $\left\{x_{n}\right\}$ in $X$ and mean $\mu$ on $\mathbb{N}$ such that

$$
\inf _{x \in X} \mu_{n} d\left(x_{n}, x\right)=0 \text {, }
$$

there exists $x_{0} \in X$ with $\mu_{n} d\left(x_{n}, x_{0}\right)=0$.

Using the above theorems, we obtain the following; see [11] and [10].

Corollary 1. Let $X$ be a metric space. Then the following are equivalent:

(i) $X$ is complete;

(ii) every weakly contractive mapping from $X$ into itself has a fixed point in $X$;

(iii) every weakly Kannan mapping from $X$ into itself has a fixed point in $X$.

Proof. (i) $\Rightarrow$ (ii) is proved in [11] and (i) $\Rightarrow$ (iii) is proved in [10]. By Theorem 1 , we have $W K_{0}(X)=W C_{1}(X) \subset W K_{1}(X) \cup W K_{2}(X)$. Since $W K_{0}(X)$ contains all Kannan mappings from $X$ into itself, we can prove (ii) $\Rightarrow$ (i) and (iii) $\Rightarrow$ (i) from Theorem 2.

Remark. We know the characterization of metric completeness by Dugundji [3]. As in the proof of Lemma 3 below, we can obtain his result from Corollary 1.

\section{ProOFs}

In this section, we give the proofs of Theorems 1 and 2 .

Before proving Theorem 1, we need some lemmas. The following lemma is essentially proved in [6]; see also [10].

Lemma 1. Let $X$ be a metric space with metric d, let $p$ be a $w$-distance on $X$ and let $f$ be a function from $X$ into $[0, \infty)$. Then a function $q$ from $X \times X$ into $[0, \infty)$ given by $q(x, y)=f(x)+p(x, y)$ for each $(x, y) \in X \times X$ is also a $w$-distance.

The following lemma is crucial in the proof of Theorem 1.

Lemma 2. Let $X$ be a metric space with metric d, let $p$ be a w-distance on $X$, let $T$ be a mapping from $X$ into itself and let $u$ be a point of $X$ such that

$$
\lim _{m, n \rightarrow \infty} p\left(T^{m} u, T^{n} u\right)=0 .
$$


Then for every $x \in X, \lim _{k} p\left(T^{k} u, x\right)$ and $\lim _{k} p\left(x, T^{k} u\right)$ exist. Moreover, let $\beta$ and $\gamma$ be functions from $X$ into $[0, \infty)$ defined by

$$
\beta(x)=\lim _{k \rightarrow \infty} p\left(T^{k} u, x\right) \quad \text { and } \quad \gamma(x)=\lim _{k \rightarrow \infty} p\left(x, T^{k} u\right) .
$$

Then the following hold:

(i) $\beta$ is lower semicontinuous on $X$;

(ii) for every $\varepsilon>0$, there exists $\delta>0$ such that $\beta(x) \leq \delta$ and $\beta(y) \leq \delta$ imply $d(x, y) \leq \varepsilon$. In particular, the set $\{x \in X: \beta(x)=0\}$ consists of at most one point;

(iii) the functions $q_{1}$ and $q_{2}$ from $X \times X$ into $[0, \infty)$ defined by

$$
q_{1}(x, y)=\beta(x)+\beta(y) \quad \text { and } \quad q_{2}(x, y)=\gamma(x)+\beta(y)
$$

are $w$-distances on $X$.

Proof. Let $x \in X$. Since

$$
\left|p\left(T^{m} u, x\right)-p\left(T^{n} u, x\right)\right| \leq \max \left\{p\left(T^{m} u, T^{n} u\right), p\left(T^{n} u, T^{m} u\right)\right\}
$$

and

$$
\left|p\left(x, T^{m} u\right)-p\left(x, T^{n} u\right)\right| \leq \max \left\{p\left(T^{m} u, T^{n} u\right), p\left(T^{n} u, T^{m} u\right)\right\}
$$

for $m, n \in \mathbb{N},\left\{p\left(T^{k} u, x\right)\right\}$ and $\left\{p\left(x, T^{k} u\right)\right\}$ are Cauchy sequences. So, $\beta$ and $\gamma$ are well-defined. We next show that $\beta$ is lower semicontinuous on $X$. Fix $x \in X$ and let $\left\{x_{n}\right\}$ be a sequence which converges to $x$. Let $\varepsilon>0$. Then there exists $k_{0} \in \mathbb{N}$ such that $p\left(T^{k_{0}} u, x\right) \geq \beta(x)-\varepsilon$ and $p\left(T^{k_{0}} u, T^{m} u\right) \leq \varepsilon$ for every $m \in \mathbb{N}$ with $m \geq k_{0}$. Fix $n \in \mathbb{N}$ and choose $k_{1} \geq k_{0}$ such that $p\left(T^{k_{1}} u, x_{n}\right) \leq \beta\left(x_{n}\right)+\varepsilon$. Then we have

$$
p\left(T^{k_{0}} u, x_{n}\right) \leq p\left(T^{k_{0}} u, T^{k_{1}} u\right)+p\left(T^{k_{1}} u, x_{n}\right) \leq \beta\left(x_{n}\right)+2 \varepsilon
$$

for every $n \in \mathbb{N}$. Hence

$$
\beta(x) \leq p\left(T^{k_{0}} u, x\right)+\varepsilon \leq \varliminf_{n \rightarrow \infty} p\left(T^{k_{0}} u, x_{n}\right)+\varepsilon \leq \varliminf_{n \rightarrow \infty} \beta\left(x_{n}\right)+3 \varepsilon .
$$

Since $\varepsilon>0$ is arbitrary, we have $\beta(x) \leq \underline{\lim }_{n} \beta\left(x_{n}\right)$. Therefore $\beta$ is lower semicontinuous on $X$. We next show (ii). Let $\varepsilon>0$ and choose $\delta>0$ such that $p(z, v) \leq 2 \delta$ and $p(z, w) \leq 2 \delta$ imply $d(v, w) \leq \varepsilon$. Suppose $\beta(x) \leq \delta$ and $\beta(y) \leq \delta$. Then there exists $k_{2} \in \mathbb{N}$ such that $p\left(T^{k_{2}} u, x\right) \leq 2 \delta$ and $p\left(T^{k_{2}} u, y\right) \leq 2 \delta$. Hence we have $d(x, y) \leq \varepsilon$. Therefore (ii) is shown. Let us prove (iii). From (i) and (ii), the function $q_{3}$ from $X \times X$ into $[0, \infty)$ defined by $q_{3}(x, y)=\beta(y)$ is $w$-distance. So, by Lemma 1 , we have $q_{1}$ and $q_{2}$ are $w$-distances on $X$. This completes the proof.

The following lemma is essentially proved in [10]. However, for the sake of completeness, we give the proof by using Lemma 2 .

Lemma 3. $W C_{1}(X) \subset W K_{0}(X)$.

Proof. Suppose $T \in W C_{1}(X)$, i.e., there exist a $w$-distance $p$ and $r \in[0,1)$ such that $p(T x, T y) \leq r p(x, y)$ for all $x, y \in X$. Fix $u \in X$. Then we have, for each $m, n \in \mathbb{N}$,

$$
p\left(T^{m} u, T^{n} u\right) \leq \frac{r^{\min \{m, n\}}}{1-r} \max \{p(u, u), p(T u, u), p(u, T u)\} .
$$


Since $0 \leq r<1$, we have $\lim _{m, n} p\left(T^{m} u, T^{n} u\right)=0$. So, by Lemma $2, \beta(x)=$ $\lim _{k} p\left(T^{k} u, x\right)$ is well-defined and $q_{1}(x, y)=\beta(x)+\beta(y)$ is a $w$-distance on $X$. From $\beta(T x) \leq r \beta(x)$ for every $x \in X$, we have

$$
q_{1}(T x, T y) \leq r(1+r)^{-1}\left(q_{1}(T x, x)+q_{1}(T y, y)\right)
$$

for all $x, y \in X$. This implies $T \in W K_{0}(X)$.

We continue studying the relations between the classes of mappings.

Lemma 4. $W K_{1}(X) \subset W C_{0}(X)$.

Proof. Suppose $T \in W K_{1}(X)$, i.e., there exist a $w$-distance $p$ and $\alpha \in[0,1 / 2)$ such that $p(T x, T y) \leq \alpha p(T x, x)+\alpha p(T y, y)$ for all $x, y \in X$. We put $r=\alpha(1-\alpha)^{-1}$. Note that $p\left(T^{2} x, T x\right) \leq r p(T x, x)$ for every $x \in X$. Fix $u \in X$. For $m, n \in \mathbb{N}$, we have

$$
p\left(T^{m} u, T^{n} u\right) \leq \alpha p\left(T^{m} u, T^{m-1} u\right)+\alpha p\left(T^{n} u, T^{n-1} u\right) \leq \alpha\left(r^{m-1}+r^{n-1}\right) p(T u, u)
$$

and hence $\lim _{m, n} p\left(T^{m} u, T^{n} u\right)=0$. So, by Lemma $2, \beta(x)=\lim _{k} p\left(T^{k} u, x\right)$ is well-defined and $q_{1}(x, y)=\beta(x)+\beta(y)$ is a $w$-distance on $X$. We next prove that $\beta(T x) \leq r \beta(x)$ for every $x \in X$. In fact, from

$$
p(T x, x) \leq p\left(T x, T^{k} u\right)+p\left(T^{k} u, x\right) \leq \alpha p(T x, x)+\alpha p\left(T^{k} u, T^{k-1} u\right)+p\left(T^{k} u, x\right),
$$

we have

$$
p\left(T^{k} u, T x\right) \leq \alpha p\left(T^{k} u, T^{k-1} u\right)+\alpha p(T x, x) \leq r p\left(T^{k} u, T^{k-1} u\right)+r p\left(T^{k} u, x\right) .
$$

Hence $\beta(T x) \leq r \beta(x)$. So we have $q_{1}(T x, T y) \leq r q_{1}(x, y)$ for all $x, y \in X$. This implies $T \in W C_{0}(X)$.

Lemma 5. $W C_{2}(X)=W K_{2}(X)$.

Proof. We first show $W C_{2}(X) \subset W K_{2}(X)$. Suppose $T \in W C_{2}(X)$, i.e., there exist a $w$-distance $p$ and $r \in[0,1)$ such that $p(T x, T y) \leq r p(y, x)$ for all $x, y \in X$. Fix $u \in X$ and $m, n \in \mathbb{N}$. If $m>n$, then

$$
\begin{aligned}
p\left(T^{m} u, T^{n} u\right)+p\left(T^{n} u, T^{m} u\right) & \leq \sum_{i=n}^{m-1}\left\{p\left(T^{i+1} u, T^{i} u\right)+p\left(T^{i} u, T^{i+1} u\right)\right\} \\
& \leq \frac{r^{n}}{1-r}\{p(T u, u)+p(u, T u)\} .
\end{aligned}
$$

If $m=n$, then $p\left(T^{m} u, T^{n} u\right) \leq r^{m} p(u, u)$. So, we have

$$
p\left(T^{m} u, T^{n} u\right) \leq \frac{r^{\min \{m, n\}}}{1-r}\{p(u, u)+p(T u, u)+p(u, T u)\}
$$

and hence $\lim _{m, n} p\left(T^{m} u, T^{n} u\right)=0$. So, by Lemma $2, \beta(x)=\lim _{k} p\left(T^{k} u, x\right)$ and $\gamma(x)=\lim _{k} p\left(x, T^{k} u\right)$ are well-defined and $q_{2}(x, y)=\gamma(x)+\beta(y)$ is a $w$-distance on $X$. From $\beta(T x) \leq r \gamma(x)$ and $\gamma(T x) \leq r \beta(x)$ for every $x \in X$, we have $q_{2}(T x, T y) \leq r(1+r)^{-1}\left(q_{2}(T x, x)+q_{2}(y, T y)\right)$ for all $x, y \in X$. This implies $T \in W K_{2}(X)$.

We next show $W K_{2}(X) \subset W C_{2}(X)$. Suppose $T \in W K_{2}(X)$, i.e., there exist a $w$-distance $p$ and $\alpha \in[0,1 / 2)$ such that $p(T x, T y) \leq \alpha p(T x, x)+\alpha p(y, T y)$ for 
all $x, y \in X$. We put $r=\alpha(1-\alpha)^{-1}$. Note that $p\left(T^{2} x, T x\right) \leq r p(x, T x)$ and $p\left(T x, T^{2} x\right) \leq r p(T x, x)$ for every $x \in X$. Fix $u \in X$. For $m, n \in \mathbb{N}$, we have

$$
\begin{aligned}
p\left(T^{m} u, T^{n} u\right) & \leq p\left(T^{m} u, T^{m-1} u\right)+p\left(T^{n-1} u, T^{n} u\right) \\
& \leq\left(r^{m-1}+r^{n-1}\right)\{p(T u, u)+p(u, T u)\}
\end{aligned}
$$

and hence $\lim _{m, n} p\left(T^{m} u, T^{n} u\right)=0$. So, by Lemma $2, \beta(x)=\lim _{k} p\left(T^{k} u, x\right)$ and $\gamma(x)=\lim _{k} p\left(x, T^{k} u\right)$ are well-defined and $q_{2}(x, y)=\gamma(x)+\beta(y)$ is a $w$-distance on $X$. We next prove that $\beta(T x) \leq r \gamma(x)$ for every $x \in X$. In fact, from

$$
p(x, T x) \leq p\left(x, T^{k} u\right)+p\left(T^{k} u, T x\right) \leq p\left(x, T^{k} u\right)+\alpha p\left(T^{k} u, T^{k-1} u\right)+\alpha p(x, T x),
$$

we have

$$
p\left(T^{k} u, T x\right) \leq \alpha p\left(T^{k} u, T^{k-1} u\right)+\alpha p(x, T x) \leq r p\left(T^{k} u, T^{k-1} u\right)+r p\left(x, T^{k} u\right) .
$$

So $\beta(T x) \leq r \gamma(x)$. Similarly, we have $\gamma(T x) \leq r \beta(x)$. Hence we have $q_{2}(T x, T y) \leq$ $r q_{2}(y, x)$ for all $x, y \in X$. This implies $T \in W C_{2}(X)$.

Now, we prove Theorem 1.

Proof of Theorem 1. It is clear that $W C_{0}(X) \subset W C_{1}(X)$ and $W K_{0}(X) \subset W K_{1}(X)$. So, by Lemmas 3 and 4 , we have

$$
W C_{0}(X)=W C_{1}(X)=W K_{0}(X)=W K_{1}(X) .
$$

Hence by Lemma 5, we obtain the desired result.

Next, we prove Theorem 2 .

Proof of Theorem 2. (i) $\Rightarrow$ (ii) was proved in [7]. We first show (ii) $\Rightarrow$ (iii). Let $\left\{x_{n}\right\}$ be a bounded sequence in $X$ and let $\mu$ be a mean on $\mathbb{N}$ such that $\inf _{x \in X} \mu_{n} d\left(x_{n}, x\right)=$ 0 . Let us define a mapping $T$ from $X$ into itself as follows. For each $x \in X$, we choose a point $T x \in X$ with $\mu_{n} d\left(x_{n}, T x\right) \leq \frac{1}{4} \mu_{n} d\left(x_{n}, x\right)$. We show that $T$ is a Kannan mapping. Let $x$ and $y$ be arbitrary points in $X$. Then

$$
\mu_{n} d\left(x_{n}, T x\right) \leq \frac{1}{4} \mu_{n} d\left(x_{n}, x\right) \leq \frac{1}{4}\left(\mu_{n} d\left(x_{n}, T x\right)+\mu_{n} d(T x, x)\right) .
$$

Hence $\mu_{n} d\left(x_{n}, T x\right) \leq \frac{1}{3} d(T x, x)$. Similarly, $\mu_{n} d\left(x_{n}, T y\right) \leq \frac{1}{3} d(T y, y)$. So we have

$$
d(T x, T y)=\mu_{n} d(T x, T y) \leq \mu_{n} d\left(x_{n}, T x\right)+\mu_{n} d\left(x_{n}, T y\right) \leq \frac{1}{3} d(T x, x)+\frac{1}{3} d(T y, y) .
$$

Hence $T$ is a Kannan mapping. From (ii), there exists a point $x_{0} \in X$ such that $T x_{0}=x_{0}$. So we have

$$
\mu_{n} d\left(x_{n}, x_{0}\right)=\mu_{n} d\left(x_{n}, T x_{0}\right) \leq \frac{1}{4} \mu_{n} d\left(x_{n}, x_{0}\right) .
$$

Hence $\mu_{n} d\left(x_{n}, x_{0}\right)=0$. This implies (iii). We next show that (iii) $\Rightarrow$ (i). Let $\left\{x_{n}\right\}$ be a Cauchy sequence in $X$ and let $\mu$ be a Banach limit. Then it is easy to see that

$$
\mu_{n} d\left(x_{n}, x\right)=\lim _{n \rightarrow \infty} d\left(x_{n}, x\right)
$$

for every $x \in X$ and

$$
\inf _{x \in X} \mu_{n} d\left(x_{n}, x\right)=0
$$


So from (iii), there exists a point $x_{0} \in X$ such that $\mu_{n} d\left(x_{n}, x_{0}\right)=0$. Hence $\lim _{n} d\left(x_{n}, x_{0}\right)=0$. Therefore $X$ is complete. This completes the proof.

Remark. (i) $\Rightarrow$ (iii) was proved in [12].

\section{Additional RESUlts}

By Theorem 1, we know $W C_{1}(X)=W C_{0}(X)=W K_{1}(X)=W K_{0}(X) \subset$ $W C_{2}(X)=W K_{2}(X)$. So it is natural to consider whether $W C_{1}(X)=W C_{2}(X)$ or not. In this section, we give two answers for this question.

Proposition 1. $W C_{1}(\mathbb{R}) \varsubsetneqq W C_{2}(\mathbb{R})$.

Proof. By the Axiom of Choice, there exists $C \subset \mathbb{R}$ such that $\operatorname{cl} C=\mathbb{R}$ and $\bigsqcup_{q \in \mathbb{Q}}(q+C)=\mathbb{R} \backslash \mathbb{Q}$, where $\operatorname{cl} C$ is the closure of $C$ and $\bigsqcup$ represents disjoint union. Define a mapping $T$ from $\mathbb{R}$ into itself by

$$
T x= \begin{cases}0, & \text { if } \quad x \in \mathbb{Q} \\ q, & \text { if } \quad x \in(q+C) \text { for some } q \in \mathbb{Q} .\end{cases}
$$

Define a $w$-distance $p$ by

$$
p(x, y)= \begin{cases}0, & \text { if } \quad x \in \mathbb{Q} \text { and } y=0, \\ 1, & \text { if } \quad x \in \mathbb{Q} \text { and } y \neq 0, \\ 2, & \text { if } \quad x \in \mathbb{R} \backslash \mathbb{Q} .\end{cases}
$$

Then we have $p(T x, T y) \leq \frac{1}{2} p(y, x)$ for all $x, y \in \mathbb{R}$. Therefore $T \in W C_{2}(\mathbb{R})$. We next show $T \notin W C_{1}(\mathbb{R})$. Suppose $T \in W C_{1}(\mathbb{R})$. Then from the proof of Lemma 3 , there exist $r \in[0,1)$ and a lower semicontinuous function $\beta$ from $\mathbb{R}$ into $[0, \infty)$ such that $\beta(T x) \leq r \beta(x)$ for every $x \in \mathbb{R}$ and the set of $\{x \in \mathbb{R}: \beta(x)=0\}$ consists of at most one point. Since $\mathbb{R}=\bigcup_{n=1}^{\infty}\{x \in \mathbb{R}: \beta(x) \leq n\}$, Baire's theorem yields that there exist $a, b \in \mathbb{R}$ such that $a<b$ and $M=\sup \beta([a, b])<\infty$. Then we obtain $M>0$ because $M=0$ implies $a=b$. Fix $q \in \mathbb{Q}$. From $\operatorname{cl}(q+C)=\mathbb{R}$, there exists $x \in(q+C) \cap[a, b]$. So, we have

$$
\beta(q)=\beta(T x) \leq r \beta(x) \leq r M .
$$

Therefore from $\operatorname{cl} \mathbb{Q}=\mathbb{R}$, we have

$$
\sup \beta(\mathbb{R})=\sup \beta(\mathbb{Q}) \leq r M<M=\sup \beta([a, b]) \leq \sup \beta(\mathbb{R}) .
$$

This is a contradiction. Hence $T \notin W C_{1}(\mathbb{R})$.

Proposition 2. If $X$ has a discrete topology, then $W C_{1}(X)=W C_{2}(X)$.

Proof. We show $W C_{2}(X) \subset W C_{1}(X)$. Suppose $T \in W C_{2}(X)$. Then from the first part of the proof of Lemma 5 , there exist $r \in[0,1)$ and a function $\beta$ from $\mathbb{R}$ into $[0, \infty)$ such that $\beta\left(T^{2} x\right) \leq r^{2} \beta(x)$ for every $x \in X$ and the following holds: For every $\varepsilon>0$, there exists $\delta>0$ such that $\beta(x) \leq \delta$ and $\beta(y) \leq \delta$ imply $d(x, y) \leq \varepsilon$. We put

$$
A=\{x \in X: \beta(x) \leq 1, \beta(T x) \leq 1\}
$$

and define a mapping $\eta$ from $X$ into $\mathbb{Z} \cup\{-\infty\}$ by

$\eta(x)= \begin{cases}-\sup \left\{n \in \mathbb{N} \cup\{0\}: \text { there exists } u \in A \text { such that } T^{n} u=x\right\}, & \text { if } x \in A, \\ \min \left\{n \in \mathbb{N}: T^{n} x \in A\right\}, & \text { if } x \notin A .\end{cases}$ 
From $\beta\left(T^{2} x\right) \leq r^{2} \beta(x), \eta$ is well-defined. Let $q$ be a function from $X \times X$ into $[0, \infty)$ defined by $q(x, y)=2^{\eta(x)}+2^{\eta(y)}$. Then $q$ is a $w$-distance on $X$. Since $\eta(T x) \leq \eta(x)-1$ for every $x \in X$, we have $q(T x, T y) \leq \frac{1}{2} q(x, y)$ for all $x, y \in X$. This implies $T \in W C_{1}(X)$.

\section{ACKNOWLEDGEMENT}

The authors wish to express their sincere thanks to the referee for giving valuable suggestions.

\section{REFERENCES}

1. S. Banach, Théorie des opérations linéaires, Monografie Mat., PWN, Warszawa, 1932.

2. J. Caristi, Fixed point theorems for mappings satisfying inwardness conditions, Trans. Amer. Math. Soc. 215 (1976), 241-251. MR 52:15132

3. J. Dugundji, Positive define functions and coincidences, Fund. Math. 90 (1976), 131-142. MR 53:4027

4. I. Ekeland, Nonconvex minimization problems, Bull. Amer. Math. Soc. 1 (1979), 443-474. MR 80h:49007

5. T. K. Hu, On a fixed-point theorem for metric spaces, Amer. Math. Monthly 74 (1967), 436-437. MR 35:1002

6. O. Kada, T. Suzuki and W. Takahashi, Nonconvex minimization theorems and fixed point theorems in complete metric spaces, Math. Japonica 44 (1996), 381-391. MR 97j:49011

7. R. Kannan, Some results on fixed points - II, Amer. Math. Monthly 76 (1969), 405-408. MR 41:2487

8. W. A. Kirk, Caristi's fixed point theorem and metric convexity, Colloquium Math. 36 (1976), 81-86. MR 55:9061

9. S. Reich, Kannan's fixed point theorem, Boll. Un. Mat. Ital. 4 (1971), 1-11. MR 46:4293

10. T. Suzuki, Several fixed point theorems in complete metric spaces, Yokohama Math. J. 44 (1997), 61-72. CMP 97:13

11. T. Suzuki and W. Takahashi, Fixed point theorems and characterizations of metric completeness, to appear in Topol. Methods Nonlinear Anal.

12. W. Takahashi, Existence theorems generalizing fixed point theorems for multivalued mappings, in Fixed Point Theory and Applications (M. A. Théra and J. B. Baillon Eds.), Pitman Research Notes in Mathematics Series 252, 397-406, John Wiley \& Sons, New York, 1991. MR 92m:54078

Faculty of Engineering, Tamagawa University, Tamagawa-Gakuen, Machida, Tokyo 194, JAPAN

E-mail address: shioji@eng.tamagawa.ac.jp

Department of Mathematical and Computing Sciences, Tokyo Institute of Technology, Ohokayama, Meguro-ku, Tokyo 152, Japan

E-mail address, T. Suzuki: tomonari@is.titech.ac.jp

E-mail address, W. Takahashi: wataru@is.titech.ac.jp 